\title{
Linearizability of Systems of Ordinary Differential Equations Obtained by Complex Symmetry Analysis
}

\author{
M. Safdar, ${ }^{1}$ Asghar Qadir, ${ }^{1}$ and S. Ali ${ }^{2,3}$ \\ ${ }^{1}$ Center for Advanced Mathematics and Physics, National University of Sciences and Technology, \\ Campus H-12, Islamabad 44000, Pakistan \\ ${ }^{2}$ School of Electrical Engineering and Computer Science, National University of Sciences and Technology, \\ Campus H-12, Islamabad 44000, Pakistan \\ ${ }^{3}$ Department of Mathematics, Brock University, St. Catherines, ON, Canada L2S 3A1 \\ Correspondence should be addressed to M. Safdar, safdar.camp@gmail.com
}

Received 16 April 2011; Accepted 22 August 2011

Academic Editor: Dane Quinn

Copyright (C) 2011 M. Safdar et al. This is an open access article distributed under the Creative Commons Attribution License, which permits unrestricted use, distribution, and reproduction in any medium, provided the original work is properly cited.

Five equivalence classes had been found for systems of two second-order ordinary differential equations, transformable to linear equations (linearizable systems) by a change of variables. An "optimal (or simplest) canonical form" of linear systems had been established to obtain the symmetry structure, namely, with 5-, 6-, 7-, 8-, and 15-dimensional Lie algebras. For those systems that arise from a scalar complex second-order ordinary differential equation, treated as a pair of real ordinary differential equations, we provide a "reduced optimal canonical form." This form yields three of the five equivalence classes of linearizable systems of two dimensions. We show that there exist 6-, 7-, and 15-dimensional algebras for these systems and illustrate our results with examples.

\section{Introduction}

Lie used algebraic symmetry properties of differential equations to extract their solutions [1-4]. One method developed was to transform the equation to linear form by changing the dependent and independent variables invertibly. Such transformations are called point transformations and the transformed equations are said to be linearized. Equations that can be so transformed are said to be linearizable. Lie proved that the necessary and sufficient condition for a scalar nonlinear ordinary differential equation (ODE) to be linearizable is that it must have eight Lie point symmetries. He exploited the fact that all scalar linear secondorder ODEs are equivalent under point transformations [5], that is, every linearizable scalar second-order ODE is reducible to the free particle equation. While the situation is not so simple for scalar linear ODEs of order $n \geq 3$, it was proved that there are three equivalence classes with $n+1, n+2$, or $n+4$ infinitesimal symmetry generators [6]. 
For linearization of systems of two nonlinear ODEs, we will first consider the equivalence of the corresponding linear systems under point transformations. Nonlinear systems of two second-order ODEs that are linearizable to systems of ODEs with constant coefficients, were proved to have three equivalence classes [7]. They have 7-,8-, or 15dimensional Lie algebras. This result was extended to those nonlinear systems which are equivalent to linear systems of ODEs with constant or variable coefficients [8]. They obtained an "optimal" canonical form of the linear systems involving three parameters, whose specific choices yielded five equivalence classes, namely, with $5-, 6-, 7-, 8-$, or 15-dimensional Lie algebras.

Geometric methods were developed to transform nonlinear systems of second-order ODEs [9-11] to a system of the free particle equations by treating them as geodesic equations and then projecting those equations down from an $m \times m$ system to an $(m-1) \times(m-1)$ system. In this process the originally homogeneous quadratically semilinear system in $m$ dimensions generically becomes a nonhomogeneous, cubically semilinear system in $(m-1)$ dimensions. When used for $m=2$ the Lie conditions for the scalar ODE are recovered precisely. The criterion for linearizability is simply that the manifold for the (projected) geodesic equations be flat. The symmetry algebra in this case is $\operatorname{sl}(n+2, \mathbb{R})$ and hence the number of generators is $n^{2}+4 n+3$. Thus for a system of two equations to be linearizable by this method it must have 15 generators. Separately, linearizability conditions have been derived for the equivalence of linearizable systems of two second-order ODEs and corresponding linear forms with $d$-dimensional Lie algebras where $d=5,6,7$, or 8 [12]. The linearization problem for two-dimensional systems of second-order ODEs has been addressed by constructing a general procedure to obtain invertible or noninvertible linearizing transformations [13]. A general solution scheme has been established to solve systems [14] which mainly consists of the reduction of the number of the dependent variables and linearization of the reduced systems. This method is known as sequential linearization and found to linearize those two-dimensional systems of second-order ODEs that are not linearizable via point transformations.

A scalar complex ODE involves two real functions of two real variables, yielding a system of two partial differential equations (PDEs) [15, 16]. By restricting the independent variable to be real we obtain a system of ODEs. Complex symmetry analysis (CSA) provides the symmetry algebra for systems of two ODEs with the help of the symmetry generators of the corresponding complex ODE. This is not a simple matter of doubling the generators for the scalar complex ODE. The inequivalence of these systems from the systems obtained by geometric means [11], has been proved [17]. Thus their symmetry structures are not the same. A two-dimensional system of second-order ODEs corresponds to a scalar complex second-order ODE if the coefficients of the system satisfy Cauchy-Riemann equations (CR-equations). We provide the full symmetry algebra of those systems that correspond to linearizable scalar complex ODEs. For this purpose we derive a reduced optimal canonical form for linear systems obtainable from a complex linear equation. We prove that this form provides three equivalence classes of linearizable systems of two second-order ODEs while there exist five linearizable classes [8] by real symmetry analysis. This difference arises due to the fact that in CSA we invoke equivalence of scalar second-order ODEs to obtain the reduced optimal form while in real symmetry analysis equivalence of linear systems of two ODEs was used to derive their optimal form. The nonlinear systems transformable to one of the three equivalence classes are characterized by complex transformations of the form

$$
T:(x, u(x)) \longrightarrow(\chi(x), U(x, u)) .
$$


Indeed, these complex transformations generate these linearizable classes of twodimensional systems. Note that not all the complex linearizing transformations for scalar complex equations provide the corresponding real transformations for systems.

The plan of the paper is as follows. In the next section we present the preliminaries for determining the symmetry structures. The third section deals with the conditions derived for systems that can be obtained by CSA. In section four we obtain the reduced optimal canonical form for systems associated with complex linear ODEs. The theory developed to classify linearizable systems of ODEs transformable to this reduced optimal form is given in the fifth section. Applications of the theory are given in the next section. The last section summarizes and discusses the work.

\section{Preliminaries}

The simplest form of a second-order equation has the maximal-dimensional algebra, $\operatorname{sl}(3, \mathbb{R})$. To discuss the equivalence of systems of two linear second-order ODEs, we need to use the following result for the equivalence of a general system of $n$ linear homogeneous secondorder ODEs with $2 n^{2}+n$ arbitrary coefficients and some canonical forms that have fewer arbitrary coefficients [18]. Any system of $n$ second-order nonhomogeneous linear ODEs

$$
\ddot{\mathbf{u}}=\mathbf{A} \dot{\mathbf{u}}+\mathbf{B u}+\mathbf{c},
$$

can be mapped invertibly to one of the following forms:

$$
\begin{aligned}
\ddot{\mathbf{v}} & =\mathrm{C} \dot{\mathbf{v}}, \\
\ddot{\mathbf{w}} & =\mathrm{Dw},
\end{aligned}
$$

where $\mathbf{A}, \mathbf{B}, \mathbf{C}, \mathbf{D}$ are $n \times n$ matrix functions, $\mathbf{u}, \mathbf{v}, \mathbf{w}, \mathbf{c}$ are vector functions, and the dot represents differentiation relative to the independent variable $t$. For a system of two secondorder ODEs $(n=2)$ there are a total of 10 coefficients for the system represented by (2.1). It is reducible to the first and second canonical forms, (2.2) and (2.3), respectively. Thus a system with 4 arbitrary coefficients of the form

$$
\begin{aligned}
& \ddot{w}_{1}=d_{11}(t) w_{1}+d_{12}(t) w_{2}, \\
& \ddot{w}_{2}=d_{21}(t) w_{1}+d_{22}(t) w_{2},
\end{aligned}
$$

can be obtained by using the equivalence of (2.1) and the counterpart of the Laguerre-Forsyth second canonical form (2.3). This result demonstrates the equivalence of systems of two ODEs having 10 and 4 arbitrary coefficients, respectively. The number of arbitrary coefficients can be further reduced to three by the change of variables [8]

$$
\tilde{y}=\frac{w_{1}}{\rho(t)}, \quad \tilde{z}=\frac{w_{2}}{\rho(t)}, \quad x=\int^{t} \rho^{-2}(s) d s,
$$

where $\rho$ satisfies

$$
\rho^{\prime \prime}-\frac{d_{11}+d_{22}}{2} \rho=0
$$


to the linear system

$$
\begin{aligned}
& \tilde{y}^{\prime \prime}=\tilde{d}_{11}(x) \tilde{y}+\tilde{d}_{12}(x) \tilde{z}, \\
& \tilde{z}^{\prime \prime}=\tilde{d}_{21}(x) \tilde{y}-\tilde{d}_{11}(x) \tilde{z},
\end{aligned}
$$

where

$$
\tilde{d}_{11}=\frac{\rho^{3}\left(d_{11}-d_{22}\right)}{2}, \quad \tilde{d}_{12}=\rho^{3} d_{12}, \quad \tilde{d}_{21}=\rho^{3} d_{21}
$$

This procedure of reduction of arbitrary coefficients for linearizable systems simplifies the classification problem enormously. System (2.7) is called the optimal canonical form for linear systems of two second-order ODEs, as it has the fewest arbitrary coefficients, namely, three.

\section{Systems of ODEs Obtainable by CSA}

Following the classical Lie procedure [19], one uses invertible point transformations

$$
X=X(x, y, z), \quad Y=Y(x, y, z), \quad Z=Z(x, y, z),
$$

to map the general system of ODEs

$$
\begin{aligned}
& y^{\prime \prime}=\Gamma_{1}\left(x, y, z, y^{\prime}, z^{\prime}\right), \\
& z^{\prime \prime}=\Gamma_{2}\left(x, y, z, y^{\prime}, z^{\prime}\right),
\end{aligned}
$$

where prime denotes differentiation with respect to $x$, to the simplest form

$$
Y^{\prime \prime}=0, \quad Z^{\prime \prime}=0,
$$

where the prime now denotes differentiation with respect to $X$ and the derivatives transform as

$$
\begin{aligned}
& Y^{\prime}=\frac{D_{x}(Y)}{D_{x}(X)}=F_{1}\left(x, y, z, y^{\prime}, z^{\prime}\right), \\
& Z^{\prime}=\frac{D_{x}(Z)}{D_{x}(X)}=F_{2}\left(x, y, z, y^{\prime}, z^{\prime}\right), \\
& Y^{\prime \prime}=\frac{D_{x}\left(F_{1}\right)}{D_{x}(X)}, \quad Z^{\prime \prime}=\frac{D_{x}\left(F_{2}\right)}{D_{x}(X)},
\end{aligned}
$$


where $D_{x}$ is the total derivative operator. The most general linearizable form of (3.2) obtainable from the transformations (3.1) is given by

$$
\begin{aligned}
y^{\prime \prime} & +\alpha_{11} y^{\prime 3}+\alpha_{12} y^{\prime 2} z^{\prime}+\alpha_{13} y^{\prime} z^{\prime 2}+\alpha_{14} z^{3}+\beta_{11} y^{\prime 2}+\beta_{12} y^{\prime} z^{\prime}+\beta_{13} z^{\prime 2} \\
& +\gamma_{11} y^{\prime}+\gamma_{12} z^{\prime}+\delta_{1}=0, \\
z^{\prime \prime}+ & \alpha_{21} y^{\prime 3}+\alpha_{22} y^{\prime 2} z^{\prime}+\alpha_{23} y^{\prime} z^{\prime 2}+\alpha_{24} z^{\prime 3}+\beta_{21} y^{\prime 2}+\beta_{22} y^{\prime} z^{\prime}+\beta_{23} z^{\prime 2} \\
& +\gamma_{21} y^{\prime}+\gamma_{22} z^{\prime}+\delta_{2}=0,
\end{aligned}
$$

where the coefficients are functions of the dependent and independent variables, which are given in the appendix. System (3.6) is the most general candidate for two second-order ODEs that may be linearizable. On the other hand CSA deals with a class of systems of the forms (3.2) in which $\Gamma_{1}$ and $\Gamma_{2}$ satisfy CR-equations. The role of CR-equations in yielding the solutions of two-dimensional systems from the solutions of complex base equations has been investigated [20]. To make a complete characterization of such systems we restate the following theorem.

Theorem 3.1. A general two-dimensional system of second-order ODEs (3.2) corresponds to a complex equation

$$
u^{\prime \prime}=\Gamma\left(x, u, u^{\prime}\right)
$$

if and only if $\Gamma_{1}$ and $\Gamma_{2}$ satisfy the CR-equations

$$
\begin{array}{cl}
\Gamma_{1, y}=\Gamma_{2, z}, & \Gamma_{1, z}=-\Gamma_{2, y}, \\
\Gamma_{1, y^{\prime}}=\Gamma_{2, z^{\prime}}, & \Gamma_{1, z^{\prime}}=-\Gamma_{2, y^{\prime}},
\end{array}
$$

where $\Gamma\left(x, u, u^{\prime}\right)=\Gamma_{1}\left(x, y, z, y^{\prime}, z^{\prime}\right)+i \Gamma_{2}\left(x, y, z, y^{\prime}, z^{\prime}\right)$.

Another candidate of linearizability of two dimensional systems obtainable from the most general form of a complex linearizable equation

$$
u^{\prime \prime}+E_{3}(x, u) u^{3}+E_{2}(x, u) u^{\prime 2}+E_{1}(x, u) u^{\prime}+E_{0}(x, u)=0,
$$

where $u$ is a complex function of the real independent variable $x$, is also cubically semilinear, that is, a system of the form

$$
\begin{aligned}
y^{\prime \prime}+ & \bar{\alpha}_{11} y^{\prime 3}-3 \bar{\alpha}_{12} y^{\prime 2} z^{\prime}-3 \bar{\alpha}_{11} y^{\prime} z^{\prime 2}+\bar{\alpha}_{12} z^{\prime 3}+\bar{\beta}_{11} y^{\prime 2}-2 \bar{\beta}_{12} y^{\prime} z^{\prime}-\bar{\beta}_{11} z^{\prime 2} \\
& +\bar{\gamma}_{11} y^{\prime}-\bar{\gamma}_{12} z^{\prime}+\bar{\delta}_{11}=0, \\
z^{\prime \prime}+ & \bar{\alpha}_{12} y^{\prime 3}+3 \bar{\alpha}_{11} y^{\prime 2} z^{\prime}-3 \bar{\alpha}_{12} y^{\prime} z^{\prime 2}-\bar{\alpha}_{11} z^{\prime 3}+\bar{\beta}_{12} y^{\prime 2}+2 \bar{\beta}_{11} y^{\prime} z^{\prime}-\bar{\beta}_{12} z^{\prime 2} \\
& +\bar{\gamma}_{12} y^{\prime}+\bar{\gamma}_{11} z^{\prime}+\bar{\delta}_{12}=0,
\end{aligned}
$$


here the coefficients $\bar{\alpha}_{1 i}, \bar{\beta}_{1 i}, \bar{\gamma}_{1 i}$, and $\bar{\delta}_{1 i}$ for $i=1,2$ are functions of $x, y$, and $z$. Clearly, the coefficients $\bar{\alpha}_{1 i}, \bar{\beta}_{1 i}, \bar{\gamma}_{1 i}$, and $\bar{\delta}_{1 i}$ satisfy the CR-equations, that is, $\bar{\alpha}_{11, y}=\bar{\alpha}_{12, z}, \bar{\alpha}_{12, y}=-\bar{\alpha}_{11, z}$ and vice versa. It is obvious that (3.9) generates a system by decomposing the complex coefficients $E_{j}$, for $j=0,1,2,3$ into real and imaginary parts

$$
E_{3}=\bar{\alpha}_{11}+i \bar{\alpha}_{12}, \quad E_{2}=\bar{\beta}_{11}+i \bar{\beta}_{12}, \quad E_{1}=\bar{\gamma}_{11}+i \bar{\gamma}_{12}, \quad E_{0}=\bar{\delta}_{11}+i \bar{\delta}_{12}
$$

where all the functions are analytic. The system of the form (3.10) is called complex linearizable as it comes from a complex linearizable ODE (3.9). In order to establish correspondence between cubically semilinear forms (3.6) and (3.10), we state the following theorem.

Theorem 3.2. A system of the form (3.6) corresponds to (3.10) if and only if coefficients of both the systems satisfy the following set of equations:

$$
\begin{array}{cc}
\alpha_{11}=-\frac{1}{3} \alpha_{13}=\frac{1}{3} \alpha_{22}=-\alpha_{24}=\bar{\alpha}_{11}, & \beta_{21}=-\frac{1}{2} \beta_{12}=-\beta_{23}=\bar{\beta}_{12}, \\
\alpha_{14}=-\frac{1}{3} \alpha_{12}=-\frac{1}{3} \alpha_{23}=\alpha_{21}=\bar{\alpha}_{12}, & \beta_{11}=\frac{1}{2} \beta_{22}=-\beta_{13}=\bar{\beta}_{11}, \\
\gamma_{11}=\gamma_{22}=\bar{\gamma}_{11}, \quad \gamma_{21}=-\gamma_{12}=\bar{\gamma}_{12}, & \delta_{1}=\bar{\delta}_{11}, \quad \delta_{2}=\bar{\delta}_{12} .
\end{array}
$$

Theorem 3.2 identifies those two-dimensional systems which are complex linearizable. It may be pointed out that the coefficients of (3.6) also satisfy CR-equations as a result of (3.12).

\section{Reduced Optimal Canonical Forms}

The simplest forms for linear systems of two second-order ODEs corresponding to complex scalar ODEs can be established by invoking the equivalence of scalar second-order linear ODEs. Consider a general linear scalar complex second-order ODE

$$
u^{\prime \prime}=\zeta_{1}(x) u^{\prime}+\zeta_{2}(x) u+\zeta_{3}(x)
$$

where prime denotes differentiation relative to $x$. As all the linear scalar second-order ODEs are equivalent, so (4.1) is equivalent to the following scalar second-order complex ODEs

$$
\begin{aligned}
& u^{\prime \prime}=\zeta_{4}(x) u^{\prime}, \\
& u^{\prime \prime}=\zeta_{5}(x) u,
\end{aligned}
$$

where all the three forms (4.1), (4.2), and (4.3) are transformable to each other. Indeed these three forms are reducible to the free particle equation. These three complex scalar linear ODEs belong to the same equivalence class, that is, all have eight Lie point symmetry generators. In this paper we prove that the systems obtainable by these forms using CSA have more than one equivalence class. To extract systems of two linear ODEs from (4.2) and (4.3) we put $u(x)=y(x)+i z(x), \zeta_{4}(x)=\alpha_{1}(x)+i \alpha_{2}(x)$ and $\zeta_{5}(x)=\alpha_{3}(x)+i \alpha_{4}(x)$, to obtain two forms of 
systems of two linear ODEs

$$
\begin{aligned}
& y^{\prime \prime}=\alpha_{1}(x) y^{\prime}-\alpha_{2}(x) z^{\prime}, \\
& z^{\prime \prime}=\alpha_{2}(x) y^{\prime}+\alpha_{1}(x) z^{\prime}, \\
& y^{\prime \prime}=\alpha_{3}(x) y-\alpha_{4}(x) z, \\
& z^{\prime \prime}=\alpha_{4}(x) y+\alpha_{3}(x) z,
\end{aligned}
$$

thus we state the following theorem.

Theorem 4.1. If a system of two second-order ODEs is linearizable via invertible complex point transformations then it can be mapped to one of the two forms (4.4) or (4.5).

Notice that here we have only two arbitrary coefficients in both the linear forms while the minimum number obtained before was three, that is, a system of the form (2.7). The reason we can reduce further is that we are dealing with the special classes of linear systems of ODEs that correspond to the scalar complex ODEs. In fact (4.5) can be reduced further by the change of variables

$$
Y=\frac{y}{\rho(x)}, \quad Z=\frac{z}{\rho(x)}, \quad X=\int^{x} \rho^{-2}(s) d s,
$$

where $\rho$ satisfies

$$
\rho^{\prime \prime}-\alpha_{3} \rho=0
$$

to

$$
\begin{gathered}
Y^{\prime \prime}=-\beta(X) Z \\
Z^{\prime \prime}=\beta(X) Y
\end{gathered}
$$

where $\beta=\rho^{3} \alpha_{4}$, and prime denotes the differentiation with respect to $X$. Hence we arrive at the following result.

Theorem 4.2. Any linear system of two second-order ODEs of the form (4.5) with two arbitrary coefficients is transformable to a simplest system of two linear ODEs (4.8) with one arbitrary coefficient via real point transformations (4.6) where (4.7) holds.

The system (4.8) provides the reduced optimal canonical form associated with complex ODEs that contain a single coefficient $\beta(x)$, which is an arbitrary function of $x$. The equivalence of systems (4.4) and (4.5) can be established via invertible point transformations which we state in the form of following theorem. 
Theorem 4.3. Two linear forms of the systems of two second-order ODEs (4.4) and (4.5) are equivalent via invertible point transformations

$$
y=M_{1}(x) y_{1}-M_{2}(x) y_{2}+y^{*}, \quad z=M_{1}(x) y_{2}+M_{2}(x) y_{1}+z^{*},
$$

of the dependent variables only, where $M_{1}(x), M_{2}(x)$ are two linearly independent solutions of

$$
\begin{aligned}
& \alpha_{1} M_{1}-\alpha_{2} M_{2}=2 M_{1}^{\prime}, \\
& \alpha_{2} M_{1}+\alpha_{1} M_{2}=2 M_{2}^{\prime},
\end{aligned}
$$

and $y^{*}, z^{*}$ are the particular solutions of (4.4).

Proof. Differentiating the set of equations (4.9) and inserting the result in the linear form (4.4), routine calculations show that under the conditions (4.10) system (4.4) can be mapped to (4.5) where

$$
\begin{aligned}
& \alpha_{3}(x)=\frac{1}{M_{1}^{2}+M_{2}^{2}}\left(M_{1}\left(\alpha_{1} M_{1}^{\prime}-\alpha_{2} M_{2}^{\prime}-M_{1}^{\prime \prime}\right)+M_{2}\left(\alpha_{1} M_{2}^{\prime}+\alpha_{2} M_{1}^{\prime}-M_{2}^{\prime \prime}\right)\right), \\
& \alpha_{4}(x)=\frac{1}{M_{1}^{2}+M_{2}^{2}}\left(M_{1}\left(\alpha_{1} M_{2}^{\prime}+\alpha_{2} M_{1}^{\prime}-M_{2}^{\prime \prime}\right)-M_{2}\left(\alpha_{1} M_{1}^{\prime}-\alpha_{2} M_{2}^{\prime}-M_{1}^{\prime \prime}\right)\right) .
\end{aligned}
$$

Thus the linear form (4.4) is reducible to (4.8).

Remark 4.4. Any nonlinear system of two second-order ODEs that is linearizable by complex methods can be mapped invertibly to a system of the form (4.8) with one coefficient which is an arbitrary function of the independent variable.

\section{Symmetry Structure of Linear Systems Obtained by CSA}

To use the reduced canonical form [21] for deriving the symmetry structure of linearizable systems associated with the complex scalar linearizable ODEs, we obtain a system of PDEs whose solution provides the symmetry generators for the corresponding linearizable systems of two second-order ODEs.

Theorem 5.1. Linearizable systems of two second-order ODEs reducible to the linear form (4.8) via invertible complex point transformations, have 6-, 7-, or 15-dimensional Lie point symmetry algebras.

Proof. The symmetry conditions provide the following set of PDEs for the system (4.8)

$$
\begin{gathered}
\xi_{, x x}=\xi_{, x y}=\xi_{, y y}=0=\eta_{1, z z}=\eta_{2, y y}, \\
\eta_{1, y y}-2 \xi_{x y}=\eta_{1, y z}-\xi_{, x z}=\eta_{2, y z}-\xi_{x y}=\eta_{2, z z}-2 \xi_{, x z}=0, \\
\xi_{, x x}-2 \eta_{1, x y}-3 z \beta(x) \xi_{, y}+y \beta(x) \xi_{, z}=\eta_{1, x z}+z \beta(x) \xi_{, z}=0, \\
\xi_{, x x}-2 \eta_{2, x z}+3 y \beta(x) \xi_{, z}-z \beta(x) \xi_{, y}=\eta_{2, x y}-y \beta(x) \xi_{, y}=0,
\end{gathered}
$$


Mathematical Problems in Engineering

$$
\begin{aligned}
& \eta_{1, x x}+\beta(x)\left(y \eta_{1, z}+2 z \xi_{, x}-z \eta_{1, y}+\eta_{2}\right)+z \beta^{\prime}(x) \xi=0, \\
& \eta_{2, x x}+\beta(x)\left(y \eta_{2, z}-2 y \xi_{, x}-z \eta_{2, y}-\eta_{1}\right)-y \beta^{\prime}(x) \xi=0 .
\end{aligned}
$$

Equations (5.3)-(5.6) involve an arbitrary function of the independent variable and its first derivatives. Using (5.1) and (5.2) we have the following solution set

$$
\begin{gathered}
\xi=\gamma_{1}(x) y+\gamma_{2}(x) z+\gamma_{3}(x), \\
\eta_{1}=\gamma_{1}^{\prime}(x) y^{2}+\gamma_{2}^{\prime}(x) y z+\gamma_{4}(x) y+\gamma_{5}(x) z+\gamma_{6}(x), \\
\eta_{2}=\gamma_{1}^{\prime}(x) y z+\gamma_{2}^{\prime}(x) z^{2}+\gamma_{7}(x) y+\gamma_{8}(x) z+\gamma_{9}(x) .
\end{gathered}
$$

Using (5.3) and (5.4), we get

$$
\beta(x) \gamma_{1}(x)=0=\beta(x) \gamma_{2}(x) .
$$

Now assuming $\beta(x)$ to be zero, nonzero constant and arbitrary function of $x$ will generate the following cases.

Case $1(\beta(x)=0)$. The set of determining equations (5.1)-(5.6) will reduce to a trivial system of PDEs

$$
\begin{gathered}
\eta_{1, x x}=\eta_{1, x z}=\eta_{1, z z}=0, \\
\eta_{2, x x}=\eta_{2, x y}=\eta_{2, y y}=0, \\
2 \xi_{, x y}-\eta_{1, y y}=0=2 \xi_{, x z}-\eta_{2, z z,} \\
\xi_{, x z}-\eta_{1, y z}=0=\xi_{, x y}-\eta_{2, y z}, \\
\xi_{, x x}-2 \eta_{1, x y}=0=\xi_{, x x}-2 \eta_{2, x z},
\end{gathered}
$$

which can be extracted classically for the system of free particle equations. Solving it we find a 15-dimensional Lie point symmetry algebra.

Case $2(\beta(x) \neq 0)$. Then (5.8) implies $\gamma_{1}(x)=\gamma_{2}(x)=0$ and (5.7) reduces to

$$
\begin{gathered}
\xi=\gamma_{3}(x), \\
\eta_{1}=\left(\frac{\gamma_{3}^{\prime}(x)}{2}+c_{3}\right) y+c_{1} z+\gamma_{6}(x), \\
\eta_{2}=c_{2} y+\left(\frac{\gamma_{3}^{\prime}(x)}{2}+c_{4}\right) z+\gamma_{9}(x) .
\end{gathered}
$$

Here three subcases arise. 
Subcase $2.1(\beta(x)$ is a non-zero constant). As equations (5.5) and (5.6) involve the derivatives of $\beta(x)$, which will now be zero, equations (5.3)-(5.6) and (5.10) yield a 7-dimensional Lie algebra. The explicit expressions of the symmetry generators involve trigonometric functions. But for a simple demonstration of the algorithm consider $\beta(x)=1$. The solution of the set of the determining equations is

$$
\begin{aligned}
\xi= & C_{1}, \\
\eta_{1}= & C_{2} y+\left(-C_{4} e^{x / \sqrt{2}}-C_{3} e^{-x / \sqrt{2}}\right) \sin \left(\frac{x}{\sqrt{2}}\right)+C_{6} e^{x / \sqrt{2}} \cos \left(\frac{x}{\sqrt{2}}\right) \\
& +C_{5} e^{-x / \sqrt{2}} \cos (x / \sqrt{2})+C_{7} z, \\
\eta_{2}= & \left(-C_{6} e^{x / \sqrt{2}}+C_{5} e^{-x / \sqrt{2}}\right) \sin \left(\frac{x}{\sqrt{2}}\right)-C_{4} e^{x / \sqrt{2}} \cos \left(\frac{x}{\sqrt{2}}\right)-C_{2} z \\
& +C_{3} e^{-x / \sqrt{2}} \cos (x / \sqrt{2})+C_{7} y .
\end{aligned}
$$

This yields a 7-dimensional symmetry algebra.

Subcase $2.2\left(\beta(x)=x^{-2}, x^{-4}\right.$, or $\left.(x+1)^{-4}\right)$. Equations (5.3)-(5.6) and (5.10) yield a 7dimensional Lie algebra. Thus the 7-dimensional algebras can be related with systems which have variable coefficients in their linear forms, apart from the linear forms with constant coefficients.

Subcase $2.3\left(\beta(x)=x^{-1}, x^{2}, x^{2} \pm C_{0}\right.$ or $\left.e^{x}\right)$. Using equations (5.3)-(5.6) and (5.10), we arrive at a 6-dimensional Lie point symmetry algebra. The explicit expressions involve special functions, for example, for $\beta(x)=x^{-1}, x^{2}, x^{2} \pm C_{0}$ we get Bessel functions. Similarly for $\beta(x)=e^{x}$ there are six symmetries, including the generators $y \partial_{y}-e^{x} z \partial_{z}, z \partial_{z}+e^{x} y \partial_{y}$. The remaining four generators come from the solution of an ODE of order four.

Thus there appear only 6-, 7-, or 15-dimensional algebras for linearizable systems of two second-order ODEs transformable to (4.8) via invertible complex point transformations. We are not investigating the remaining two linear forms (4.4) and (4.5), because these are transformable to system (4.8), that is, all these forms have the same symmetry structures. Now we verify that the linear forms with Lie algebras of dimensions 6 or 7 are also obtainable from (2.7). For instance, take the coefficients in (2.7) as non-zero constants, $\tilde{d}_{11}(x)=a_{0}$, $\tilde{d}_{12}(x)=b_{0}$, and $\tilde{d}_{21}(x)=c_{0}$, where

$$
a_{0}^{2}+b_{0} c_{0} \neq 0
$$

The corresponding system has 7-dimensional algebra which is the same as the linear form (4.8). Moreover the 8-dimensional symmetry algebra was extracted [8] by assuming

$$
a_{0}^{2}+b_{0} c_{0}=0
$$

Such linear forms cannot be obtained from (4.8). These two examples explain why a 7dimensional algebra can be obtained from (4.8), but a linear form with an 8-dimensional algebra is not obtainable from it. 
To prove these observations consider arbitrary point transformations of the form

$$
\tilde{y}=a(x) y+b(x) z, \quad \tilde{z}=c(x) y+d(x) z .
$$

Case a. If $a(x)=a_{0}, b(x)=b_{0}, c(x)=c_{0}$, and $d(x)=d_{0}$ are constants then (5.14) implies

$$
\begin{aligned}
& \widetilde{y}^{\prime \prime}=a_{0} y^{\prime \prime}+b_{0} z^{\prime \prime}, \\
& \widetilde{z}^{\prime \prime}=c_{0} y^{\prime \prime}+d_{0} z^{\prime \prime} .
\end{aligned}
$$

Using (2.7) and (4.5) in the above equation we find

$$
\begin{aligned}
\left(a_{0} d_{0}-b_{0} c_{0}\right) y^{\prime \prime}= & \left(\left(a_{0} d_{0}+b_{0} c_{0}\right) \tilde{d}_{11}(x)+c_{0} d_{0} \tilde{d}_{12}(x)-a_{0} b_{0} \tilde{d}_{21}(x)\right) y \\
& +\left(2 b_{0} d_{0} \tilde{d}_{11}(x)+d_{0}^{2} \tilde{d}_{12}(x)-b_{0}^{2} \tilde{d}_{21}(x)\right) z \\
\left(a_{0} d_{0}-b_{0} c_{0}\right) z^{\prime \prime}=( & \left.\left(a_{0} d_{0}+b_{0} c_{0}\right) \tilde{d}_{11}(x)+c_{0} d_{0} \tilde{d}_{12}(x)-a_{0} b_{0} \tilde{d}_{21}(x)\right) z \\
& +\left(2 a_{0} c_{0} \tilde{d}_{11}(x)+c_{0}^{2} \tilde{d}_{12}(x)-a_{0}^{2} \tilde{d}_{21}(x)\right) y
\end{aligned}
$$

where $a_{0} d_{0}-b_{0} c_{0} \neq 0$. Using (4.5), (5.16), and the linear independence of the $\tilde{d}^{\prime}$ s, gives

$$
\begin{gathered}
a_{0} b_{0}=c_{0} d_{0}=0, \\
a_{0}^{2}-b_{0}^{2}=c_{0}^{2}-d_{0}^{2}=0, \\
a_{0} d_{0}+b_{0} c_{0}=a_{0} c_{0}-b_{0} d_{0}=0,
\end{gathered}
$$

which has a solution $a_{0}=b_{0}=c_{0}=d_{0}=0$, which is inconsistent with (5.16) because the requirement was $a_{0} d_{0}-b_{0} c_{0} \neq 0$.

Case $b$. If $a(x), b(x), c(x)$, and $d(x)$ are arbitrary functions of $x$ then

$$
\begin{aligned}
& \tilde{y}^{\prime \prime}=a(x) y^{\prime \prime}+b(x) z^{\prime \prime}+a^{\prime \prime}(x) y+b^{\prime \prime}(x) z+2 a^{\prime}(x) y^{\prime}+2 b^{\prime}(x) z^{\prime}, \\
& \tilde{z}^{\prime \prime}=c(x) y^{\prime \prime}+d(x) z^{\prime \prime}+c^{\prime \prime}(x) y+d^{\prime \prime}(x) z+2 c^{\prime}(x) y^{\prime}+2 d^{\prime}(x) z^{\prime} .
\end{aligned}
$$

Thus we obtain

$$
\begin{aligned}
(a d-b c) y^{\prime \prime}= & {\left[(a d+b c) \tilde{d}_{11}+c d \tilde{d}_{12}-a b \tilde{d}_{21}-a^{\prime \prime} d+c^{\prime \prime} b\right] y } \\
& +\left(2 b d \tilde{d}_{11}+d^{2} \tilde{d}_{12}-b^{2} \tilde{d}_{21}-b^{\prime \prime} d+d^{\prime \prime} b\right) z-2 d\left(a^{\prime} y^{\prime}+b^{\prime} z^{\prime}\right)+2 b\left(c^{\prime} y^{\prime}+d^{\prime} z^{\prime}\right), \\
(a d-b c) z^{\prime \prime}= & \left(2 a c \tilde{d}_{11}+c^{2} \tilde{d}_{12}-a^{2} \tilde{d}_{21}-a^{\prime \prime} c+c^{\prime \prime} a\right) y \\
& +\left[(a d+b c) \tilde{d}_{11}+c d \tilde{d}_{12}-a b \tilde{d}_{21}-b^{\prime \prime} c+d^{\prime \prime} a\right] z-2 c\left(a^{\prime} y^{\prime}+b^{\prime} z^{\prime}\right)+2 a\left(c^{\prime} y^{\prime}+d^{\prime} z^{\prime}\right) .
\end{aligned}
$$


Comparing the coefficients as before and using the linear independence of $\tilde{d}^{\prime}$ s we obtain

$$
a^{\prime}(x)=b^{\prime}(x)=c^{\prime}(x)=d^{\prime}(x)=0,
$$

which implies that it reduces to a system of the form (5.16), which leaves us again with the same result. Thus we have the following theorem.

Theorem 5.2. The linear forms for systems of two second-order ODEs obtainable by real symmetry analysis with 5- or 8-dimensional algebras are not transformable to (4.5) by invertible point transformations.

Before presenting some illustrative applications of the theory developed we refine Theorem 5.1 by using Theorem 5.2 to make the following remark.

Remark 5.3. There are only 6-, 7-, or 15-dimensional algebras for linearizable systems obtainable by scalar complex linearizable ODEs, that is, there are no 5- or 8-dimensional Lie point symmetry algebras for such systems.

\section{Applications}

Consider a system of nonhomogeneous geodesic-type differential equations

$$
\begin{gathered}
y^{\prime \prime}+y^{\prime 2}-z^{\prime 2}=\Omega_{1}\left(x, y, z, y^{\prime}, z^{\prime}\right), \\
z^{\prime \prime}+2 y^{\prime} z^{\prime}=\Omega_{2}\left(x, y, z, y^{\prime}, z^{\prime}\right),
\end{gathered}
$$

where $\Omega_{1}$ and $\Omega_{1}$ are linear functions of the dependent variables and their derivatives. This system corresponds to a complex scalar equation

$$
u^{\prime \prime}+u^{\prime 2}=\Omega\left(x, u, u^{\prime}\right),
$$

which is either transformable to the free particle equation or one of the linear forms (4.1)(4.3), by means of the complex transformations

$$
x=x(x), \quad U(x)=e^{u} .
$$

Which are further transformable to the free particle equation by utilizing another set of invertible complex point transformations. Generally, the system (6.1) is transformable to a system of the free particle equations or a linear system of the form

$$
\begin{aligned}
& Y^{\prime \prime}=\tilde{\Omega}_{1}\left(X, Y, Z, Y^{\prime}, Z^{\prime}\right)-\tilde{\Omega}_{2}\left(X, Y, Z, Y^{\prime}, Z^{\prime}\right), \\
& Z^{\prime \prime}=\widetilde{\Omega}_{2}\left(X, Y, Z, Y^{\prime}, Z^{\prime}\right)+\widetilde{\Omega}_{1}\left(X, Y, Z, Y^{\prime}, Z^{\prime}\right) .
\end{aligned}
$$

Here $\widetilde{\Omega}_{1}$ and $\widetilde{\Omega}_{2}$ are linear functions of the dependent variables and their derivatives, via an invertible change of variables obtainable from (6.3). The linear form (6.4) can be mapped to a maximally symmetric system if and only if there exist some invertible complex transformations of the form (6.3), otherwise these forms cannot be reduced further. 
This is the reason why we obtain three equivalence classes, namely, with 6-, 7-, and 15dimensional algebras for systems corresponding to linearizable complex equations with only one equivalence class. We first consider an example of a nonlinear system that admits a 15-dimensional algebra which can be mapped to the free particle system using (6.3). Then we consider four applications to nonlinear systems of quadratically semilinear ODEs transformable to (6.4) via (6.3) that are not further reducible to the free particle system.

(1) Consider (6.1) with

$$
\begin{aligned}
& \Omega_{1}=-\frac{2}{x} y^{\prime}, \\
& \Omega_{2}=-\frac{2}{x} z^{\prime},
\end{aligned}
$$

it admits a 15-dimensional algebra. The real linearizing transformations

$$
X(x)=\frac{1}{x}, \quad Y=e^{y} \cos (z), \quad Z=e^{y} \sin (z),
$$

obtainable from the complex transformations (6.3) with $U(X)=Y(X)+i Z(X)$, map the above nonlinear system to $Y^{\prime \prime}=0, Z^{\prime \prime}=0$. Moreover, the solution of (6.5) corresponds to the solution of the corresponding complex equation

$$
u^{\prime \prime}+u^{\prime 2}+\frac{2}{x} u^{\prime}=0
$$

(2) Now consider $\Omega_{1}$ and $\Omega_{2}$ to be linear functions of the first derivatives $y^{\prime}, z^{\prime}$, that is, system (6.1) with

$$
\begin{aligned}
& \Omega_{1}=c_{1} y^{\prime}-c_{2} z^{\prime}, \\
& \Omega_{2}=c_{2} y^{\prime}+c_{1} z^{\prime},
\end{aligned}
$$

which admits a 7-dimensional algebra, provided both $c_{1}$ and $c_{2}$ are not simultaneously zero. It is associated with the complex equation

$$
u^{\prime \prime}+u^{\prime 2}-c u^{\prime}=0
$$

Using the transformations (6.3) to generate the real transformations

$$
X(x)=x, \quad Y=e^{y} \cos (z), \quad Z=e^{y} \sin (z),
$$

which map the nonlinear system to a linear system of the form (4.4), that is,

$$
\begin{aligned}
& Y^{\prime \prime}=c_{1} Y^{\prime}-c_{2} Z^{\prime}, \\
& Z^{\prime \prime}=c_{2} Y^{\prime}+c_{1} Z^{\prime},
\end{aligned}
$$

which also has a 7-dimensional symmetry algebra and corresponds to

$$
U^{\prime \prime}-c U^{\prime}=0 \text {. }
$$


All the linear second-order ODEs are transformable to the free particle equation thus we can invertibly transform the above equation to $\tilde{U}^{\prime \prime}=0$, using

$$
(x(x), U) \longrightarrow\left(\tilde{X}=\alpha+\beta e^{c X(x)}, \tilde{U}=U\right)
$$

where $\alpha, \beta$, and $c$ are complex. But these complex transformations can not generate real transformations to reduce the corresponding system (6.11) to a maximally symmetric system.

(3) A system with a 6-dimensional Lie algebra is obtainable from (6.1) by introducing a linear function of $x$ in the above coefficients, that is,

$$
\begin{aligned}
& \Omega_{1}=(1+x)\left(c_{1} y^{\prime}-c_{2} z^{\prime}\right), \\
& \Omega_{2}=(1+x)\left(c_{2} y^{\prime}+c_{1} z^{\prime}\right),
\end{aligned}
$$

in (6.1), then the same transformations (6.10) converts the above system into a linear system

$$
\begin{aligned}
& Y^{\prime \prime}=(1+\chi)\left(c_{1} Y^{\prime}-c_{2} Z^{\prime}\right), \\
& Z^{\prime \prime}=(1+\chi)\left(c_{2} Y^{\prime}+c_{1} Z^{\prime}\right),
\end{aligned}
$$

where both systems (6.14) and (6.15) are in agreement on the dimensions (i.e., six) of their symmetry algebras. Again, the above system is a special case of the linear system (4.4).

(4) If we choose $\Omega_{1}=c_{1}, \Omega_{2}=c_{2}$, where $c_{i}(i=1,2)$ are non-zero constants, then under the same real transformations (6.10), the nonlinear system (6.1) takes the form

$$
\begin{aligned}
& Y^{\prime \prime}=c_{1} Y-c_{2} Z, \\
& Z^{\prime \prime}=c_{2} Y+c_{1} Z .
\end{aligned}
$$

\section{Conclusion}

The classification of linearizable systems of two second-order ODEs was obtained by using the equivalence properties of systems of two linear second-order ODEs [8]. The "optimal canonical form" of the corresponding linear systems of two second-order ODEs, to which a linearizable system could be mapped, is crucial. This canonical form used invertible transformations, the invertibility of these mappings insuring that the symmetry structure is preserved. That optimal canonical form of the linear systems of two second-order ODEs led to five linearizable classes with respect to Lie point symmetry algebras with dimensions 5,6 , 7,8 , and 15 .

Systems of two second-order ODEs appearing in CSA correspond to some scalar complex second-order ODE. We proved the existence of a reduced optimal canonical form for such linear systems of two ODEs. This reduced canonical form provided three equivalence classes, namely, with 6-, 7-, or 15-dimensional point symmetry algebras. Two cases are eliminated in the theory of complex symmetries: those of 5 and 8-dimensional algebras. The systems corresponding to a complex linearized scalar ODE involve one parameter which can only cover three possibilities: (a) it is zero, (b) it is a non-zero constant, and (c) it is a nonconstant function. The nonexistence of 5- and 8-dimensional algebras for the linear forms 
appearing due to CSA has been proved by showing that these forms are not equivalent to those provided by the real symmetry approach for systems [8] with 5 and 8 generators.

Work is in progress [20] to find complex methods of solving a class of 2-dimensional nonlinearizable systems of second-order ODEs. It is also obtainable from the linearizable scalar complex second-order ODEs, which are transformable to the free particle equation via an invertible change of the dependent and independent variables of the form

$$
x=x(x, u), \quad U(x)=U(x, u) .
$$

Notice that these transformations are different from (6.3). The real transformations corresponding to the complex transformations above cannot be used to linearize the real system. But the linearizability of the complex scalar equations can be used to provide solutions for the corresponding systems.

One might wonder how the procedures developed can be extended to odd dimensional systems of equations. To obtain a $2 n$-dimensional system we can take an $n$-dimensional system, regard it as complex and split it. This method will not work for odd dimensions. An extension of the procedure has been developed [22] of using the splitting procedure iteratively starting with a scalar base equation. Among others, this gave a 3-dimensional system of ODEs. The procedure could be used by increasing the number of iterations or starting with a higher dimensional system and using a second iteration, to obtain any dimensional system—even or odd.

\section{Appendix}

Inserting $F_{1}\left(x, y, z, y^{\prime}, z^{\prime}\right)$ and $F_{2}\left(x, y, z, y^{\prime}, z^{\prime}\right)$ from (3.4) into (3.5) we obtain

$$
\begin{aligned}
& \frac{D_{x}(X) \cdot D_{x}^{2}(Y)-D_{x}(Y) \cdot D_{x}^{2}(X)}{\left(D_{x}(X)\right)^{3}}=0 \\
& \frac{D_{x}(X) \cdot D_{x}^{2}(Z)-D_{x}(Z) \cdot D_{x}^{2}(X)}{\left(D_{x}(X)\right)^{3}}=0 .
\end{aligned}
$$

Substituting

$$
\begin{gathered}
D_{x}(X)=X_{x}+y^{\prime} X_{y}+z^{\prime} X_{z \prime} \\
D_{x}^{2}(X)=X_{x x}+2 y^{\prime} X_{x y}+2 z^{\prime} X_{x z}+y^{\prime 2} X_{y y}+2 y^{\prime} z^{\prime} X_{y z}+z^{\prime 2} X_{z z}+y^{\prime \prime} X_{y}+z^{\prime \prime} X_{z \prime}
\end{gathered}
$$

and similar expressions for $D_{x}(Y), D_{x}(Z), D_{x}^{2}(Y)$, and $D_{x}^{2}(Z)$ in (A.1), yields

$$
\begin{aligned}
& \bar{\alpha}_{1} y^{\prime \prime}+\bar{\alpha}_{2} z^{\prime \prime}+\bar{\beta}_{1} y^{\prime 3}+\bar{\beta}_{2} y^{\prime 2} z^{\prime}+\bar{\beta}_{3} y^{\prime} z^{2}+\bar{\beta}_{4} z^{\prime 3}+\bar{\gamma}_{1} y^{\prime 2}+\bar{\gamma}_{2} y^{\prime} z^{\prime}+\bar{\gamma}_{3} z^{\prime 2} \\
& +\bar{\delta}_{1} y^{\prime}+\bar{\delta}_{2} z^{\prime}+\bar{\epsilon}_{1}=0, \\
& \bar{\alpha}_{3} y^{\prime \prime}+\bar{\alpha}_{4} z^{\prime \prime}+\bar{\beta}_{5} y^{\prime 3}+\bar{\beta}_{6} y^{\prime 2} z^{\prime}+\bar{\beta}_{7} y^{\prime} z^{\prime 2}+\bar{\beta}_{8} z^{\prime 3}+\bar{\gamma}_{4} y^{\prime 2}+\bar{\gamma}_{5} y^{\prime} z^{\prime}+\bar{\gamma}_{6} z^{\prime 2} \\
& +\bar{\delta}_{3} y^{\prime}+\bar{\delta}_{4} z^{\prime}+\bar{\epsilon}_{2}=0 .
\end{aligned}
$$


The coefficients of the above system of ODEs are

$$
\begin{gathered}
\bar{\alpha}_{1}=X_{x} Y_{y}-Y_{x} X_{y}+z^{\prime}\left(X_{z} Y_{y}-Y_{z} X_{y}\right), \quad \bar{\alpha}_{2}=X_{x} Y_{z}-Y_{x} X_{z}+y^{\prime}\left(X_{y} Y_{z}-Y_{y} X_{z}\right), \\
\bar{\alpha}_{3}=X_{x} Z_{y}-Z_{x} X_{y}+z^{\prime}\left(X_{z} Z_{y}-Z_{z} X_{y}\right), \quad \bar{\alpha}_{4}=X_{x} Z_{z}-Z_{x} X_{z}+y^{\prime}\left(X_{y} Z_{z}-Z_{y} X_{z}\right), \\
\bar{\beta}_{1}=X_{y} Y_{y y}-Y_{y} X_{y y}, \quad \bar{\beta}_{2}=X_{z} Y_{y y}-Y_{z} X_{y y}+2\left(X_{y} Y_{y z}-Y_{y} X_{y z}\right), \\
\bar{\beta}_{3}=X_{y} Y_{z z}-Y_{y} X_{z z}+2\left(X_{z} Y_{y z}-Y_{z} X_{y z}\right), \quad \bar{\beta}_{4}=X_{z} Y_{z z}-Y_{z} X_{z z}, \\
\bar{\beta}_{5}=X_{y} Z_{y y}-Z_{y} X_{y y}, \quad \bar{\beta}_{6}=X_{z} Z_{y y}-Z_{z} X_{y y}+2\left(X_{y} Z_{y z}-Z_{y} X_{y z}\right), \\
\bar{\beta}_{7}=X_{y} Z_{z z}-Z_{y} X_{z z}+2\left(X_{z} Z_{y z}-Z_{z} X_{y z}\right), \quad \bar{\beta}_{8}=X_{z} Z_{z z}-Z_{z} X_{z z}, \\
\bar{\gamma}_{1}=X_{x} Y_{y y}-Y_{x} X_{y y}+2\left(X_{y} Y_{x y}-Y_{y} X_{x y}\right), \\
\bar{\gamma}_{2}=2\left[X_{x} Y_{y z}+X_{y} Y_{x z}+X_{z} Y_{x y}-\left(Y_{x} X_{y z}+Y_{y} X_{x z}+Y_{z} X_{x y}\right)\right], \\
\bar{\gamma}_{3}=X_{x} Y_{z z}-Y_{x} X_{z z}+2\left(X_{z} Y_{x z}-Y_{z} X_{x z}\right), \\
\bar{\gamma}_{4}=X_{x} Z_{y y}-Z_{x} X_{y y}+2\left(X_{y} Z_{x y}-Z_{y} X_{x y}\right), \\
\bar{\gamma}_{5}=2\left[X_{x} Z_{y z}+X_{y} Z_{x z}+X_{z} Z_{x y}-\left(Z_{x} X_{y z}+Z_{y} X_{x z}+Z_{z} X_{x y}\right)\right], \\
\bar{\gamma}_{6}=X_{x} Z_{z z}-Z_{x} X_{z z}+2\left(X_{z} Z_{x z}-Z_{z} X_{x z}\right), \\
\bar{\delta}_{1}=X_{y} Y_{x x}-Y_{y} X_{x x}+2\left(X_{x} Y_{x y}-Y_{x} X_{x y}\right), \\
\bar{\delta}_{2}=X_{z} Y_{x x}-Y_{z} X_{x x}+2\left(X_{x} Y_{x z}-Y_{x} X_{x z}\right), \\
\bar{\delta}_{3}=X_{y} Z_{x x}-Z_{y} X_{x x}+2\left(X_{x} Z_{x y}-Z_{x} X_{x y}\right), \\
\bar{\delta}_{4}=X_{z} Z_{x x}-Z_{z} X_{x x}+2\left(X_{x} Z_{x z}-Z_{x} X_{x z}\right), \\
\bar{\epsilon}_{1}=X_{x} Y_{x x}-Y_{x} X_{x x}, \quad \bar{\epsilon}_{2}=X_{x} Z_{x x}-Z_{x} X_{x x} .
\end{gathered}
$$

System (A.3) yields a system of the form (3.6) with the following coefficients:

$$
\begin{array}{rrr}
\alpha_{11}=\tau_{1}\left(\bar{\alpha}_{4} \bar{\beta}_{1}-\bar{\alpha}_{2} \bar{\beta}_{5}\right), & \alpha_{12}=\tau_{1}\left(\bar{\alpha}_{4} \bar{\beta}_{2}-\bar{\alpha}_{2} \bar{\beta}_{6}\right), & \alpha_{13}=\tau_{1}\left(\bar{\alpha}_{4} \bar{\beta}_{3}-\bar{\alpha}_{2} \bar{\beta}_{7}\right), \\
\alpha_{14}=\tau_{1}\left(\bar{\alpha}_{4} \bar{\beta}_{4}-\bar{\alpha}_{2} \bar{\beta}_{8}\right), & \alpha_{21}=\tau_{2}\left(\bar{\alpha}_{3} \bar{\beta}_{1}-\bar{\alpha}_{1} \bar{\beta}_{5}\right), & \alpha_{22}=\tau_{2}\left(\bar{\alpha}_{3} \bar{\beta}_{2}-\bar{\alpha}_{1} \bar{\beta}_{6}\right), \\
\alpha_{23}=\tau_{2}\left(\bar{\alpha}_{3} \bar{\beta}_{3}-\bar{\alpha}_{1} \bar{\beta}_{7}\right), \quad \alpha_{24}=\tau_{2}\left(\bar{\alpha}_{3} \bar{\beta}_{4}-\bar{\alpha}_{1} \bar{\beta}_{8}\right), \\
\beta_{11}=\tau_{1}\left(\bar{\alpha}_{4} \bar{\gamma}_{1}-\bar{\alpha}_{2} \bar{\gamma}_{4}\right), & \beta_{12}=\tau_{1}\left(\bar{\alpha}_{4} \bar{\gamma}_{2}-\bar{\alpha}_{2} \bar{\gamma}_{5}\right), & \beta_{13}=\tau_{1}\left(\bar{\alpha}_{4} \bar{\gamma}_{3}-\bar{\alpha}_{2} \bar{\gamma}_{6}\right), \\
\beta_{21}=\tau_{2}\left(\bar{\alpha}_{3} \bar{\gamma}_{1}-\bar{\alpha}_{1} \bar{\gamma}_{4}\right), & \beta_{22}=\tau_{2}\left(\bar{\alpha}_{3} \bar{\gamma}_{2}-\bar{\alpha}_{1} \bar{\gamma}_{5}\right), & \beta_{23}=\tau_{2}\left(\bar{\alpha}_{2} \bar{\gamma}_{3}-\bar{\alpha}_{1} \bar{\gamma}_{6}\right), \\
\gamma_{11}=\tau_{1}\left(\bar{\alpha}_{4} \bar{\delta}_{1}-\bar{\alpha}_{2} \bar{\delta}_{3}\right), & \gamma_{12}=\tau_{1}\left(\bar{\alpha}_{4} \bar{\delta}_{2}-\bar{\alpha}_{2} \bar{\delta}_{4}\right), & \gamma_{21}=\tau_{2}\left(\bar{\alpha}_{3} \bar{\delta}_{1}-\bar{\alpha}_{1} \bar{\delta}_{3}\right), \\
\gamma_{22}=\tau_{2}\left(\bar{\alpha}_{3} \bar{\delta}_{2}-\bar{\alpha}_{1} \bar{\delta}_{4}\right), & \delta_{1}=\tau_{1}\left(\bar{\alpha}_{4} \bar{\epsilon}_{1}-\bar{\alpha}_{2} \bar{\epsilon}_{2}\right), & \delta_{2}=\tau_{2}\left(\bar{\alpha}_{3} \bar{\epsilon}_{1}-\bar{\alpha}_{1} \bar{\epsilon}_{2}\right),
\end{array}
$$

where $\tau_{1}=-\tau_{2}=\bar{\alpha}_{1} \bar{\alpha}_{4}-\bar{\alpha}_{2} \bar{\alpha}_{3}$. 


\section{Acknowledgments}

The authors are grateful to Fazal Mahomed for useful comments and discussion on this paper. M. Safader is most grateful to NUST for providing financial support.

\section{References}

[1] S. Lie, "Klassifikation und integration von gewönlichen differentialgleichungenzwischen $x, y$, die eine gruppe von transformationen gestaten," Archiv der Mathematik, vol. VIII-IX, p. 187, 1883.

[2] S. Lie, Lectures on Differential Equations with Known Infinitesimal Transformations, Teubner, Leipzig, Germany, 1891, (in German, Lie's Lectures, by G. Sheffers).

[3] S. Lie, Theorie der Transformationsgruppen I, II and III, Teubner, Leipzig, Germany, 1888, (reprinted by Chelsea Publishing, New York, NY, USA, 1970).

[4] S. Lie, Vorlesungen Ü ber Differentialgleichungen mit Bekannten Infinitesimalen Transformationen, Teubner, Leipzig, Germany, 1891, (reprinted by Chelsea Publishing, New York, NY, USA, 1967).

[5] F. M. Mahomed, "Symmetry group classification of ordinary differential equations: survey of some results," Mathematical Methods in the Applied Sciences, vol. 30, no. 16, pp. 1995-2012, 2007.

[6] F. M. Mahomed and P. G. L. Leach, "Symmetry Lie algebras of $n$th order ordinary differential equations," Journal of Mathematical Analysis and Applications, vol. 151, no. 1, pp. 80-107, 1990.

[7] V. M. Gorringe and P. G. L. Leach, "Lie point symmetries for systems of second order linear ordinary differential equations," Quaestiones Mathematicae, vol. 11, no. 1, pp. 95-117, 1988.

[8] C. Wafo Soh and F. M. Mahomed, "Symmetry breaking for a system of two linear second-order ordinary differential equations," Nonlinear Dynamics, vol. 22, no. 1, pp. 121-133, 2000.

[9] A. V. Aminova and N. A. M. Aminov, "Projective geometry of systems of differential equations: general conceptions," Tensor-New Series, vol. 62, no. 1, pp. 65-86, 2000.

[10] F. M. Mahomed and A. Qadir, "Linearization criteria for a system of second-order quadratically semilinear ordinary differential equations," Nonlinear Dynamics, vol. 48, no. 4, pp. 417-422, 2007.

[11] F. M. Mahomed and A. Qadir, "Invariant linearization criteria for systems of cubically semi-linear second-order ordinary differential equations," Journal of Nonlinear Mathematical Physics, vol. 16, pp. 1-16, 2009.

[12] Y. Y. Bagderina, "Linearization criteria for a system of two second-order ordinary differential equations," Journal of Physics, A, vol. 43, no. 46, pp. 465201-465215, 2010.

[13] V. K. Chandrasekar, M. Senthilvelan, and M. Lakshmanan, "On the complete integrability and linearization of nonlinear ordinary differential equations. V. Linearization of coupled second-order equations," Proceedings of the Royal Society of London, Series A, vol. 465, no. 2108, pp. 2369-2389, 2009.

[14] S. Sookmee and S. V. Meleshko, "Conditions for linearization of a projectable system of two secondorder ordinary differential equations," Journal of Physics, A, vol. 41, no. 40, pp. 402001-402008, 2008.

[15] S. Ali, F. M. Mahomed, and A. Qadir, "Linearizability criteria for systems of two second-order differential equations by complex methods," Nonlinear Dynamics. In press.

[16] S. Ali, F. M. Mahomed, and A. Qadir, "Complex Lie symmetries for scalar second-order ordinary differential equations," Nonlinear Analysis-Real World Applications, vol. 10, no. 6, pp. 3335-3344, 2009.

[17] M. Safdar, A. Qadir, and S. Ali, "Inequivalence of classes of linearizable systems of cubically semilinear ordinary differential equations obtained by real and complex symmetry analysis," Mathematical and Computational Applications, vol. 16, no. 4, pp. 923-934, 2011.

[18] C. W. Soh and F. M. Mahomed, "Linearization criteria for a system of second-order ordinary differential equations," International Journal of Non-Linear Mechanics, vol. 36, no. 4, pp. 671-677, 2001.

[19] J. Merker, "Characterization of the Newtonian free particle system in $m \geq 2$ dependent variables," Acta Applicandae Mathematicae, vol. 92, no. 2, pp. 125-207, 2006.

[20] M. Safdar, S. Ali, and A. Qadir, "Symmetry solutions of two-dimensional systems not solvable by symmetry analysis," arXiv:1104.3837.

[21] H. Stephani, Differential Equations: Their Solutions Using Symmetries, Cambridge University Press, Cambridge, UK, 1996.

[22] F. M. Mahomed and A. Qadir, "Higher dimensional systems of differential equations obtained by iterative use of complex methods," (preprint of DECMA, CAM, University of Witwatersrand, 2011). 


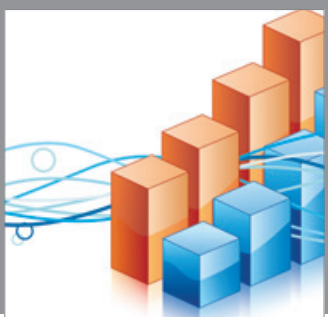

Advances in

Operations Research

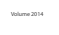

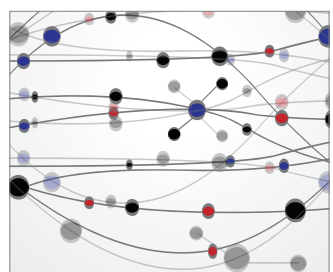

\section{The Scientific} World Journal
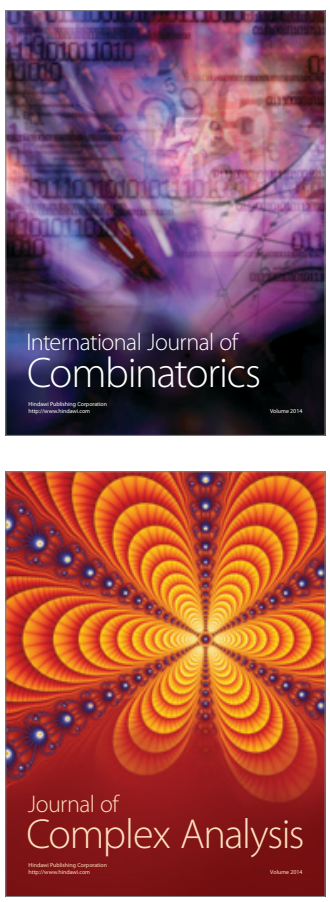

International Journal of

Mathematics and

Mathematical

Sciences
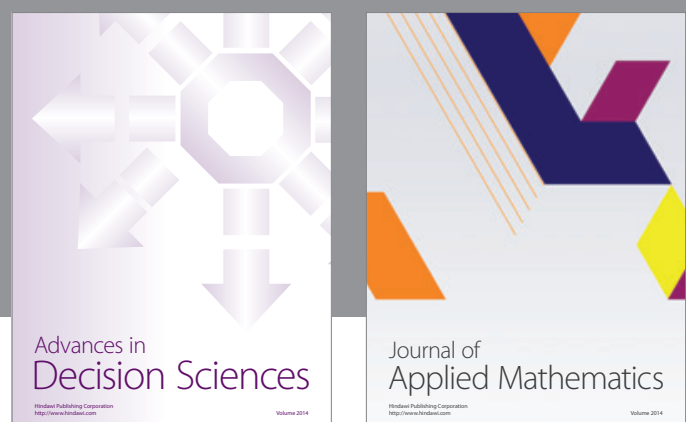

Journal of

Applied Mathematics
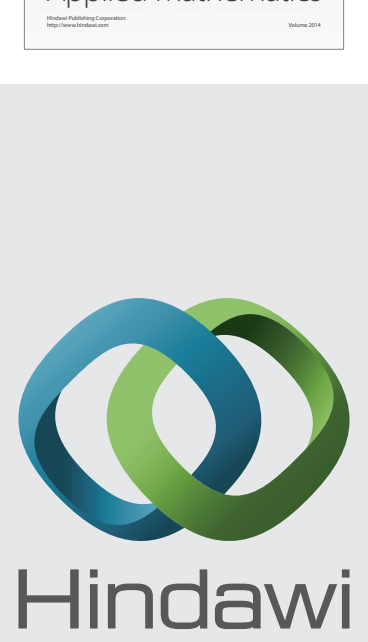

Submit your manuscripts at http://www.hindawi.com
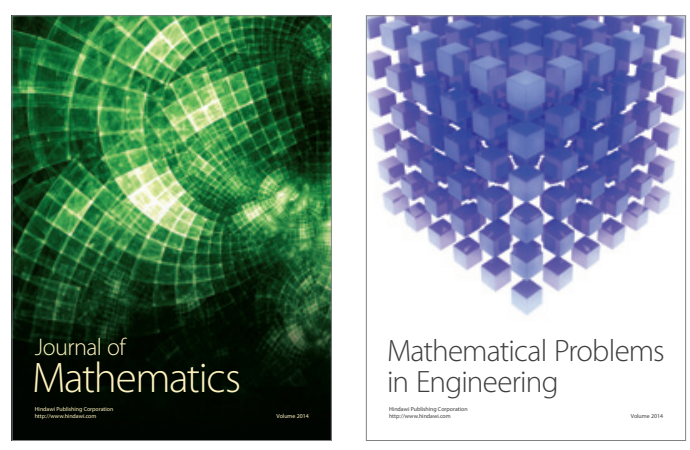

Mathematical Problems in Engineering
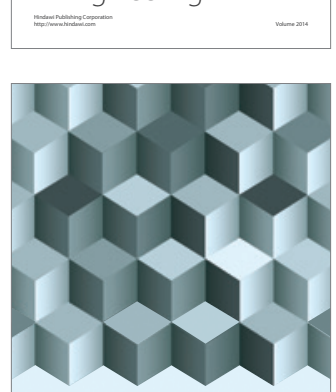

Journal of

Function Spaces
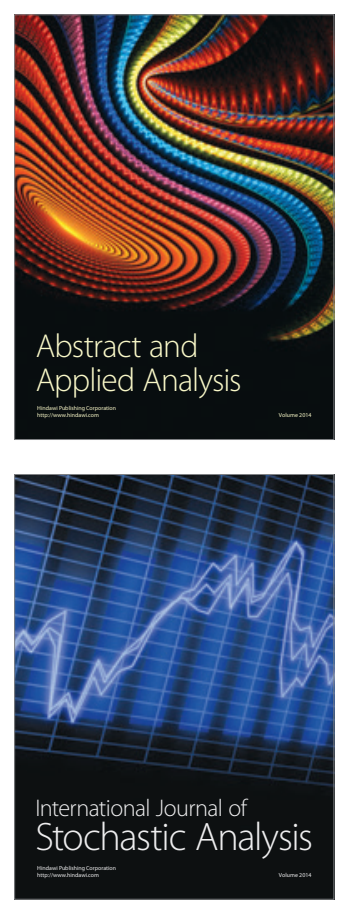

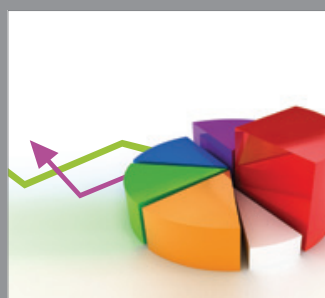

ournal of

Probability and Statistics

Promensencen
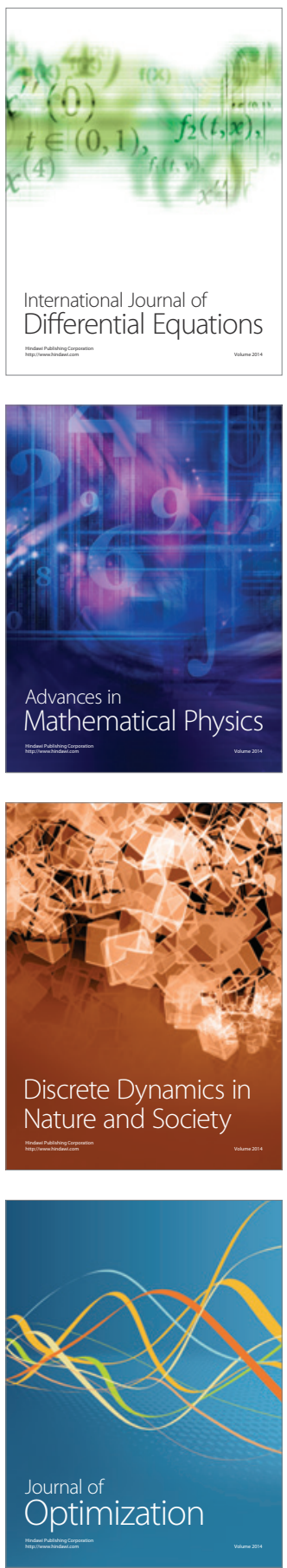